\title{
Constraining the location of rapid gamma-ray flares in the flat spectrum radio quasar $3 C 273$
}

\author{
B. Rani ${ }^{1, \star}$, B. Lott $^{2}$, T. P. Krichbaum ${ }^{1}$, L. Fuhrmann ${ }^{1}$, and J. A. Zensus ${ }^{1}$ \\ 1 Max-Planck-Institut für Radioastronomie (MPIfR), Auf dem Hügel 69, 53121 Bonn, Germany \\ e-mail: brani@mpifr-bonn.mpg.de \\ ${ }^{2}$ Université Bordeaux 1, CNRS/IN2p3, Centre d’Études Nucléaires de Bordeaux Gradignan, 33175 Gradignan, France
}

Received 8 March 2013 / Accepted 2 July 2013

\begin{abstract}
We present a $\gamma$-ray photon flux and spectral variability study of the flat-spectrum radio quasar 3C 273 over a rapid flaring activity period between September 2009 to April 2010. Five major flares were observed in the source during this period. The most rapid flare observed in the source has a flux doubling time of $1.1 \mathrm{hr}$. The rapid $\gamma$-ray flares allow us to constrain the location and size of the $\gamma$-ray emission region in the source. The $\gamma \gamma$-opacity constrains the Doppler factor $\delta_{\gamma} \geq 10$ for the highest energy ( $\left.15 \mathrm{GeV}\right)$ photon observed by the Fermi-Large Area Telescope (LAT). Causality arguments constrain the size of the emission region to $1.6 \times 10^{15} \mathrm{~cm}$. The $\gamma$-ray spectra measured over this period show clear deviations from a simple power law with a break in the 1-2 GeV energy range. We discuss possible explanations for the origin of the $\gamma$-ray spectral breaks. Our study suggests that the $\gamma$-ray emission region in 3C 273 is located within the broad line region $(<1.6 \mathrm{pc})$. The spectral behavior and temporal characteristics of the individual flares indicate the presence of multiple shock scenarios at the base of the jet.
\end{abstract}

Key words. gamma rays: galaxies - galaxies: active - quasars: individual: 3C 273

\section{Introduction}

The $\gamma$-ray bright blazar 3C 273 is classified as a flat spectrum radio quasar (FSRQ) at a redshift $z=0.158$ (Strauss et al. 1992). It was the first discovered quasar (Schmidt 1963), and the first extragalactic source detected in $\gamma$-ray band, by COS-B (Swanenburg et al. 1978). The source is categorized as a low synchrotron peaked blazar (LSP) by Abdo et al. (2010a). The broadband spectrum, the correlation among multi-frequency flares, and the VLBI jet kinematics of the source were extensively studied in the past (e.g., Krichbaum et al. 2001; Rani et al. 2010, 2011; Soldi et al. 2008; Savolainen et al. 2010; Abdo et al. $2010 \mathrm{e}$, and references therein).

After the first detection in $\gamma$-rays by COS-B, 3C 273 was also observed by EGRET in 1999 (3EG J1229+0210 in Hartman et al. 1999) with an average flux of $F(E>100 \mathrm{MeV})=$ $0.18 \times 10^{-6} \mathrm{ph} \mathrm{cm}^{-2} \mathrm{~s}^{-1}$. Krichbaum et al. (2001) noticed a correlation between jet component ejection and EGRET $\gamma$-ray flux variations, which constrained the location of the $\gamma$-ray emission region to $\sim 2000$ Schwarzschild radii $(<0.2 \mathrm{pc})$ from the central black hole $(\mathrm{BH})$. The Fermi-Large Area Telescope (LAT) detected the source in the $\gamma$-ray band from the beginning of observations in 2008 (Abdo et al. 2010a). The source was observed in a bright outburst phase at the beginning of July 2009. The first flaring event lasted for $\sim 10$ days in August 2009 (Bastieri 2009). The source exhibited another $\gamma$-ray outburst in September 2009 with two bright flares from September 15 to 19, 2009 and from September 20 to 23, 2009. Abdo et al. (2010e) discussed the

* Member of the International Max Planck Research School (IMPRS) for Astronomy and Astrophysics at the Universities of Bonn and Cologne. flare characteristics and spectral behavior during this outburst period. Before the brightest $\gamma$-ray flare was observed in 3C 454.3 in December 2009 (Escande \& Tanaka 2009; Abdo et al. 2011), 3C 273 was the brightest extragalactic non $\gamma$-ray burst source observed by Fermi-LAT. Although the $\gamma$-ray spectrum of 3C 273 integrated over the first six months of LAT observations showed a significant break around $1.5 \mathrm{GeV}$ (Abdo et al. 2010d), the spectra integrated over shorter periods of time in September 2009 did not provide indication for such curvature (Abdo et al. 2010e).

The second flare during September 20 to 30, 2009 displayed a first short peak and was followed by a period of high-activity indicating either a series of substructures or a long decay tail (Abdo et al. 2010e). The source continued the rapid flaring activity until April 2010 and later was observed in a quiescent state, which is still continuing. We refer to the flaring interval between August 2009 to April 2010 as the high-activity period. Thanks to the high flux during August 2009 to April 2010, we are able to obtain light curves with good statistics using a time binning of only $3 \mathrm{~h}$, allowing us to investigate the evolution of these outbursts. The focus of this study is to probe the location and size of the $\gamma$-ray emission region in 3C 273 by investigating its intra-day variability and associated spectral changes in the $\gamma$-ray band.

This paper is structured as follows. Section 2 provides a brief description of observations and data reduction. In Sect. 3, we report our results. We discuss our results in Sect. 4. Conclusions are given in Sect. 5.

\section{Observations and data reduction}

The $\gamma$-ray flux and spectral variations of the source were investigated using the Fermi-LAT data. The $\gamma$-ray data employed here 
were collected during a time period between JD $=2454683$ (August 04, 2008) to 2455889 (November 23, 2011) in survey mode by Fermi-LAT. We analyzed the LAT data using the standard ScienceTools (software version v9.23.1) and instrument response functions P7V6 ${ }^{1}$. Photons in the Source event class were selected for this analysis. We selected $\gamma$-ray photons with zenith angles less than $100^{\circ}$ to greatly reduce contamination from $\gamma$-rays produced by cosmic-ray interactions in the upper atmosphere. The diffuse emission from our Galaxy was modeled using a spatial model (gal_2yearp7v6_v0.fits) that was refined by the Fermi-LAT data taken during the first two years of operation. The extragalactic diffuse and residual instrumental backgrounds were modeled as an isotropic component (isotropic_p7v6source.txt), and were provided with the data analysis tools. We analyzed a region of interest of $10^{\circ}$ in radius, centered at the position of the $\gamma$-ray source associated with 3C 273 using a maximum-likelihood algorithm ${ }^{2}$ (Mattox et al. 1996). In the model for the $\gamma$-ray emission from the region, we included all the 30 sources within $10^{\circ}$ with their model parameters fixed to their catalog values except for 3C 279 and MG1 J123931+0443. The other sources are reported as being not significantly variable in the 2FGL catalog (see Nolan et al. 2012). For 3C 279 and MG1 J123931+0443, we set all model parameters free.

We investigated the source variability by producing light curves by likelihood analysis with different time binnings $(3 \mathrm{~h}$, 6 h, 12 h, 1 day, and 1 week) and over different energy ranges ( $E>100 \mathrm{MeV}, E<1 \mathrm{GeV}$, and $E>1 \mathrm{GeV}$ ). The light curves were produced by modeling the spectra for each time bin and energy range by a simple power law that was sufficient for the relatively short time ranges considered here. Also, the statistical uncertainties on the power law (PL) indices were smaller than those obtained from broken power law (BPL) fits.

We also computed photon flux light curves above the decorrelation energy $E_{0}$ (Lott et al. 2012) that minimizes the correlations between integrated photon flux and photon index $(\Gamma)$. Over the course of $\sim 3$ years of observations, we find $E_{0}=$ $167 \mathrm{MeV}$. We generated the constant uncertainty (15\%) light curve above $E_{0}$ through the adaptive binning method following Lott et al. (2012). The estimated systematic uncertainty on the flux is $10 \%$ at $100 \mathrm{MeV}, 5 \%$ at $500 \mathrm{MeV}$, and $20 \%$ at $10 \mathrm{GeV}$ (Ackermann et al. 2012).

We performed the spectral analysis by fitting the $\gamma$-ray spectra with multiple models over the whole energy range covered by Fermi-LAT above $100 \mathrm{MeV}$. The different spectral forms are simple power law [PL, $N(E)=N_{0}(E)^{-\Gamma}, N_{0}$ : prefactor, and $\Gamma$ : power law index], smoothed broken power law [SBPL, $N(E)=N_{0} E^{-\Gamma_{1}}\left(1+\left(E / E_{\text {break }}\right)^{-\left(\Gamma_{1}-\Gamma_{2}\right) / b}\right)^{-b}, \Gamma_{1}, \Gamma_{2}$ : the two power law indices, and $E_{\mathrm{Break}}$ : break energy, $b$ : smoothing parameters set to 0.1$]$, and a $\log$-parabola function $[\log \mathrm{P}, N(E)=$ $N_{0}\left(E / E_{\mathrm{P}}\right)^{-\left(\alpha+\beta \log \left(E / E_{\mathrm{P}}\right)\right.}, \alpha$ : power law index, $\beta$ : index parameter that characterizes the spectral curvature, and $E_{\mathrm{P}}$ : the peak energy fixed at $1 \mathrm{GeV}$ ]. We also examined the spectral behavior over the whole energy range with a simple PL model that we fitted over equally spaced logarithmic energy bins, with $\Gamma$ kept constant and equal to the value obtained by fitting over the whole energy range.

\footnotetext{
1 http://fermi.gsfc.nasa.gov/ssc/data/analysis/ scitools/overview.html

2 http://fermi.gsfc.nasa.gov/ssc/data/analysis/ scitools/likelihood_tutorial.html
}

\section{Analysis and results}

\subsection{Temporal characteristics}

\subsubsection{Light curves}

The source was exhibiting significant flux variability throughout the whole Fermi mission (Abdo et al. 2010b). Figure 1a displays the weekly averaged flux $F_{100}(F[E>100 \mathrm{MeV}]$ in units of $10^{-6} \mathrm{ph} \mathrm{cm}^{-2} \mathrm{~s}^{-1}$ ) light curve of the source. A sequence of flares is observed in the source between $\mathrm{JD}^{\prime 3}=1042$ to 1320 (August 2009 to April 2010) and no substantial flux variability has been observed since then. The source seems to have been in a quiescent state since April 2010, which is still continuing. The high-activity period can be seen in the box in Fig. 1. Figure 1b shows the one-day averaged $F_{100}$ light curve over this period. Five major flares labeled A to E were observed in the source during this period (see Fig. 1b). Interestingly, the major flares were separated from each other by roughly 50 days. The high flux level during the flaring periods allowed us to investigate the evolution of outbursts with a temporal resolution as fine as $3 \mathrm{~h}$ (corresponding to one sky scan of the LAT).

Figure 1a shows the arrival time distribution of $\gamma$-ray photons with energies greater than $10 \mathrm{GeV}$. During the $\sim 3$ years of observations, the highest energy photon associated with $3 \mathrm{C} 273$ was detected at $\mathrm{JD}^{\prime}=1151$ with an energy of $15.4 \mathrm{GeV}$. This photon is converted in the front section of the LAT tracker, where the angular resolution is best (Ackermann et al. 2012). The reconstructed arrival direction of the photon is $0.07^{\circ}$ away from 3 C 273. Based on our model fit of the epoch which contains the highest-energy photon, we find the probability that the photon was associated with 3C 273 (as opposed to all other sources in the model including the diffuse emission and nearby point sources) is $99.98 \%$, or $>3 \sigma$. Interestingly, the highest energy photon was observed during the flaring period.

Flare A had a duration of $\sim 12$ days with a peak flux of $\sim 5.1 \times$ $10^{-6} \mathrm{ph} \mathrm{cm}^{-2} \mathrm{~s}^{-1}$. Flare B consisted of two main sub-flares, each one having a total duration of $\sim 2$ days and a peak flux of $\sim 12 \times$ $10^{-6} \mathrm{ph} \mathrm{cm}^{-2} \mathrm{~s}^{-1}$. The first sub-flare showed a nice smooth time profile, suggesting that it is well resolved; the second sub-flare, after a first short peak, shows a series of high spikes that indicate substructures in a long decay tail. A detailed study of flux and spectral variations of these two outbursts is given in Abdo et al. (2010e). Flare $\mathrm{C}$ was recorded $\sim 50$ days later than flare B, and was followed by an extremely rapid flare $\mathrm{D}$, and a number of sub-flares as flare E.

We also investigated the evolution of these flares with finer time resolution depending upon the $\gamma$-ray brightness of the source. The light curves for $F_{100}$, with $3 \mathrm{~h}$ and $12 \mathrm{~h}$ time binning for the individual flares, are shown in Fig. 2. A fit consisting of a slowly varying background and rapid sub-flaring components is performed for each individual flare. The slowly varying background is roughly approximated by a flux value = $0.42 \times 10^{-6} \mathrm{ph} \mathrm{cm}^{-2} \mathrm{~s}^{-1}$. Each component is fitted by a function of the form

$F(t)=2 F_{0}\left[\mathrm{e}^{\left(t_{0}-t\right) / T_{\mathrm{r}}}+\mathrm{e}^{\left(t-t_{0}\right) / T_{\mathrm{f}}}\right]^{-1}$,

where $T_{r}$ and $T_{f}$ are the rise and decay times, respectively, and $F_{0}$ is the flux at $t_{0}$ representing approximately the flare amplitude. The solid curves in Fig. 2 represent the fitted flare components and the fitted parameters for each sub-component are given in Table 1. The doubling times of the individual flares is given in

$\mathrm{JD}^{\prime}=\mathrm{JD}-2454000$ 


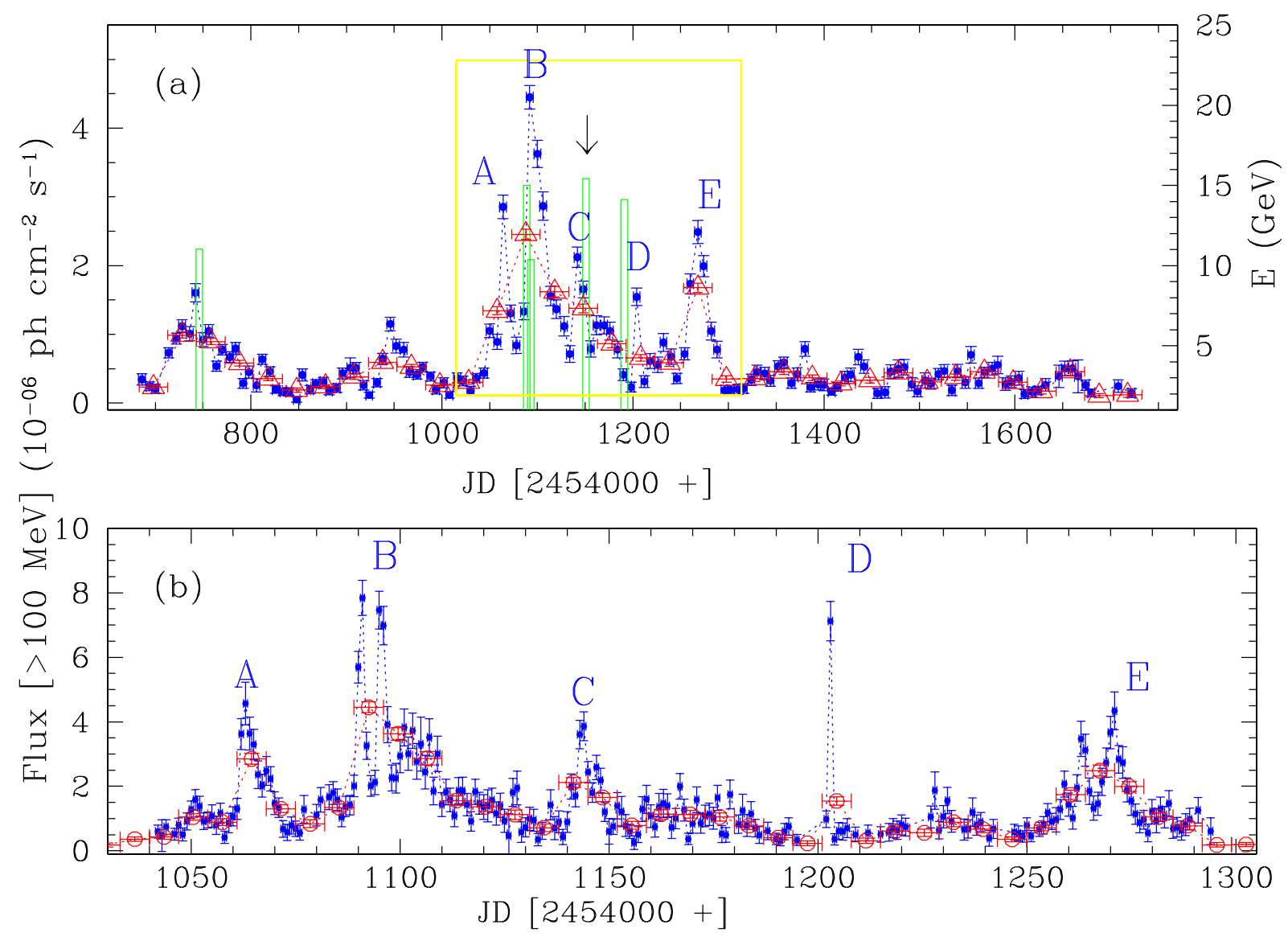

Fig. 1. a) Weekly (blue circles) and monthly (red open symbols) averaged flux ( $E>100 \mathrm{MeV}$ ) light curves of $3 \mathrm{C} 273$ measured by Fermi-LAT since its launch. The green histogram represents the arrival time distribution of $E>10 \mathrm{GeV}$ photons associated with 3C 273. The yellow box represents the high $\gamma$-ray flux activity period. b) One-day averaged light curve of the source over the high-activity period within the box shown at the top of the figure.

the last column of Table 1. We note that the flares have similar halving times because of comparable rise and decay timescales.

The short duration of flare $\mathrm{D}$, with timescales similar to the orbital period of Fermi ( $96 \mathrm{~min}$ ), makes the standard analysis, where the photons are binned in time, inappropriate for a detailed temporal characterization of the flare. A different, unbinned (in the time domain) method taking into account the strongly varying rate of accumulation of exposure associated with the survey mode was employed instead for this purpose. In this method, based on the same log-likelihood approach as described in Lott et al. (2012), a time-dependent function $f(t, E)=F(t) \times N(E)$ where $F(t)$ is given in Eq. (1) and $N(E)$ is a power law distribution (neglecting any spectral changes during the flare), was fitted to the unbinned data. The results are plotted in Fig. 3, showing from top to bottom the exposure rate, the comparison between the predicted source-photon detection rate and that estimated from the data, and the flux light curve. The parameters for $F(t)$ are given in Table 1 .

During the first component of flare D the source brightens to $F_{100} \sim 12$ with a flux doubling time of $1.1 \mathrm{~h}$. The second component of flare D has a peak flux value $F_{100} \sim 7$ with a flux doubling time of $1.5 \mathrm{~h}$. During flare D, the Moon was close to the region of interest of $3 \mathrm{C} 273$. We therefore checked for the possible contribution from the Moon. We found that the Moon was closest to the source on January 07, 2010 with an angular distance of $11^{\circ}$, which is larger than the region of interest of the source, and therefore unlikely to cause any significant contamination in the observed rapid flux variations.
Flare A shows essentially equal rise ( $\sim 1.81$ day) and decay timescales ( 1.79 day), which implies that flare $\mathrm{A}$ has a symmetric temporal profile. In contrast to flare A, the components of flare B are characterized by decay times ( 2.50 day) longer than the rise time ( $\sim 0.50$ day; Abdo et al. 2010e). For flare $\mathrm{C}$, the components have a symmetric profile except the middle sub-component (see Table 1). Both the components of flare D have relatively symmetric evolution. All the components of flare $\mathrm{E}$ are characterized by symmetric temporal profiles. Therefore, most of the flares have symmetric profiles except flare B.

\subsubsection{Flux variations at different energy bands}

The flux variations below and above $1 \mathrm{GeV}$ energy during the high-activity period are shown in Fig. 4. The flux variations below $1 \mathrm{GeV}$ seem to be more pronounced, but we note that the characteristic decay timescales of the individual flares are similar for the $F_{E<1 \mathrm{GeV}}$ and $F_{E>1 \mathrm{GeV}}$ light curves using $12 \mathrm{~h}$ time binning. Even with a finer temporal resolution of $6 \mathrm{~h}$ for the brightest flare $\mathrm{B}$ (component 1 and 2), we find that the decay timescales for the $F_{E<1 \mathrm{GeV}}$ and $F_{E>1 \mathrm{GeV}}$ light curves are not statistically different. The rise and decay timescales, the peak flux value and the respective time of peak of the flare for the $6 \mathrm{~h}$ binned light curves of these flares at different energy scales are given in Table 2.

To search for a possible time lag and to quantify the correlation among the $\gamma$-ray flux variations below and above $1 \mathrm{GeV}$, we compute the discrete cross-correlation function (DCF) of the two light curves ( $E$ below and above $1 \mathrm{GeV}$ ) following the method 
Table 1. Fitted parameters of flares.

\begin{tabular}{lcccccc}
\hline \hline $\begin{array}{l}\text { Main- } \\
\text { flare }\end{array}$ & $\begin{array}{c}\text { Sub- } \\
\text { flare }\end{array}$ & $\begin{array}{c}T_{\mathrm{r}} \\
\text { (day) }\end{array}$ & $\begin{array}{c}T_{\mathrm{f}} \\
\text { (day) }\end{array}$ & $\begin{array}{c}t_{0} \\
\mathrm{JD}^{\prime}\end{array}$ & $\begin{array}{c}F_{0} \\
10^{-6} \mathrm{ph} \mathrm{cm}^{-2} \mathrm{~s}^{-1}\end{array}$ & $\begin{array}{c}\text { Doubling } \\
\text { Time (h) }\end{array}$ \\
\hline $\mathrm{C}$ & 1 & $0.34 \pm 0.12$ & $0.39 \pm 0.13$ & $1141.52 \pm 0.11$ & $2.31 \pm 0.13$ & 5.60 \\
& 2 & $0.56 \pm 0.13$ & $1.01 \pm 0.10$ & $1143.71 \pm 0.23$ & $4.11 \pm 0.12$ & 9.30 \\
& 3 & $0.68 \pm 0.12$ & $0.87 \pm 0.18$ & $1147.61 \pm 0.64$ & $2.20 \pm 0.13$ & 11.31 \\
$\mathrm{D}^{b}$ & 1 & $0.07 \pm 0.02$ & $0.08 \pm 0.02$ & $1203.31 \pm 0.04$ & $12.30 \pm 2.83$ & 1.16 \\
& 2 & $0.09 \pm 0.04$ & $0.07 \pm 0.02$ & $1203.83 \pm 0.05$ & $7.41 \pm 3.09$ & 1.54 \\
$\mathrm{E}$ & 1 & $0.79 \pm 0.13$ & $0.53 \pm 0.11$ & $1259.22 \pm 0.24$ & $2.13 \pm 0.10$ & 13.10 \\
& 2 & $1.16 \pm 0.16$ & $1.10 \pm 0.21$ & $1263.63 \pm 0.35$ & $3.43 \pm 0.09$ & 19.32 \\
& 3 & $1.47 \pm 0.13$ & $1.68 \pm 0.18$ & $1270.72 \pm 0.31$ & $4.14 \pm 0.12$ & 24.43 \\
\hline
\end{tabular}

Notes. ${ }^{(a)}$ Doubling time $=T_{\mathrm{r}} \times \ln 2 .^{\left({ }^{(b)}\right.}$ In estimating the photon detection rate from the data, the relative fluxes of the source and background were taken from the binned-analysis results. This estimated rate is not strongly dependent on these relative fluxes, however, because of the different spatial distributions of the source and background components.

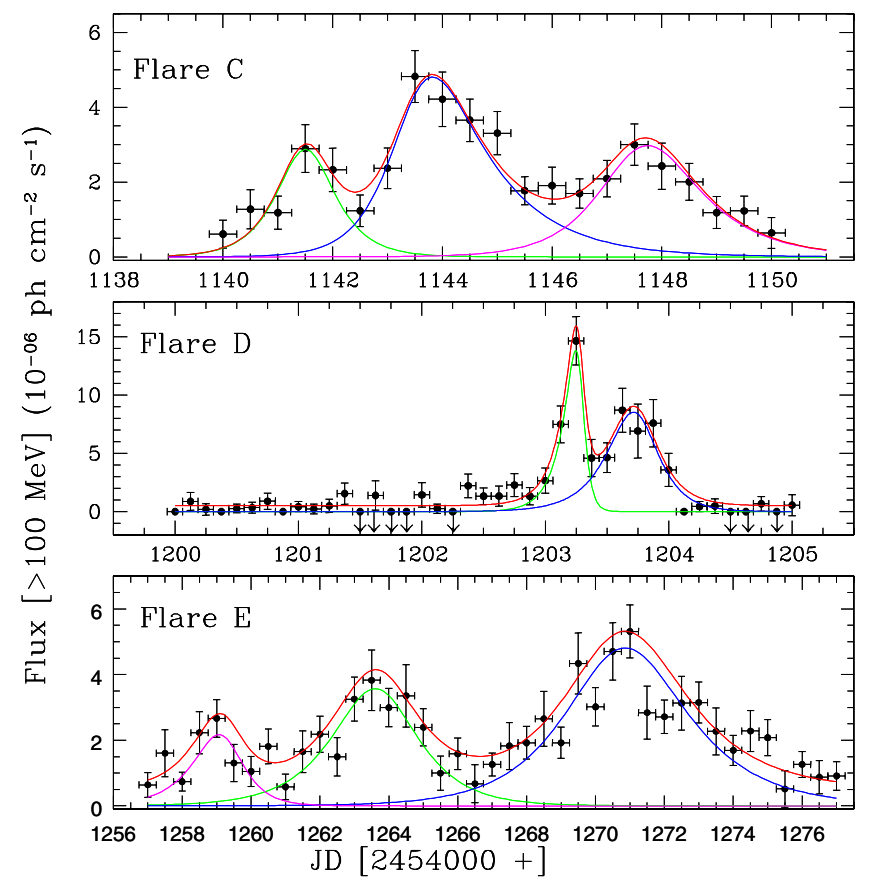

Fig. 2. Light curves of $3 \mathrm{C} 273$ above $100 \mathrm{MeV}$ with time binning of $12 \mathrm{~h}$ (flare $\mathrm{C}$ and $\mathrm{E}$ ) and $3 \mathrm{~h}$ (flare $\mathrm{D}$ ). The lines are the fitted sub-flaring components (see Table 1).

described by Edelson \& Krolik (1988). The 12 h binned DCF analysis curve (Fig. 5) shows a peak $(D C F=0.82 \pm 0.21)$ at a time lag of $0.00 \pm 0.25$ days. This confirms the existence of a significant correlation between the flux variations below and above $1 \mathrm{GeV}$ with zero time delay. Because of limited statistics for the more finely binned light curves, we cannot claim any time delay shorter than our binning interval of $12 \mathrm{~h}$.

\subsubsection{Photon index variations}

We investigated the flux variations above the de-correlation energy $\left(E_{0}\right)$ that minimizes the correlations between integrated photon flux and photon index. We generated the constant uncertainty (15\%) light curve above $E_{0}$ using the adaptive binning method (Lott et al. 2012). Figure 6 shows the photon flux and index variations during the high-activity period between $\mathrm{JD}^{\prime}=$ 1050 to 1320 at $E>E_{0}$. The different flaring activity periods of the source are separated by vertical columns. We note substantial variations in photon spectral index during different modes of flux

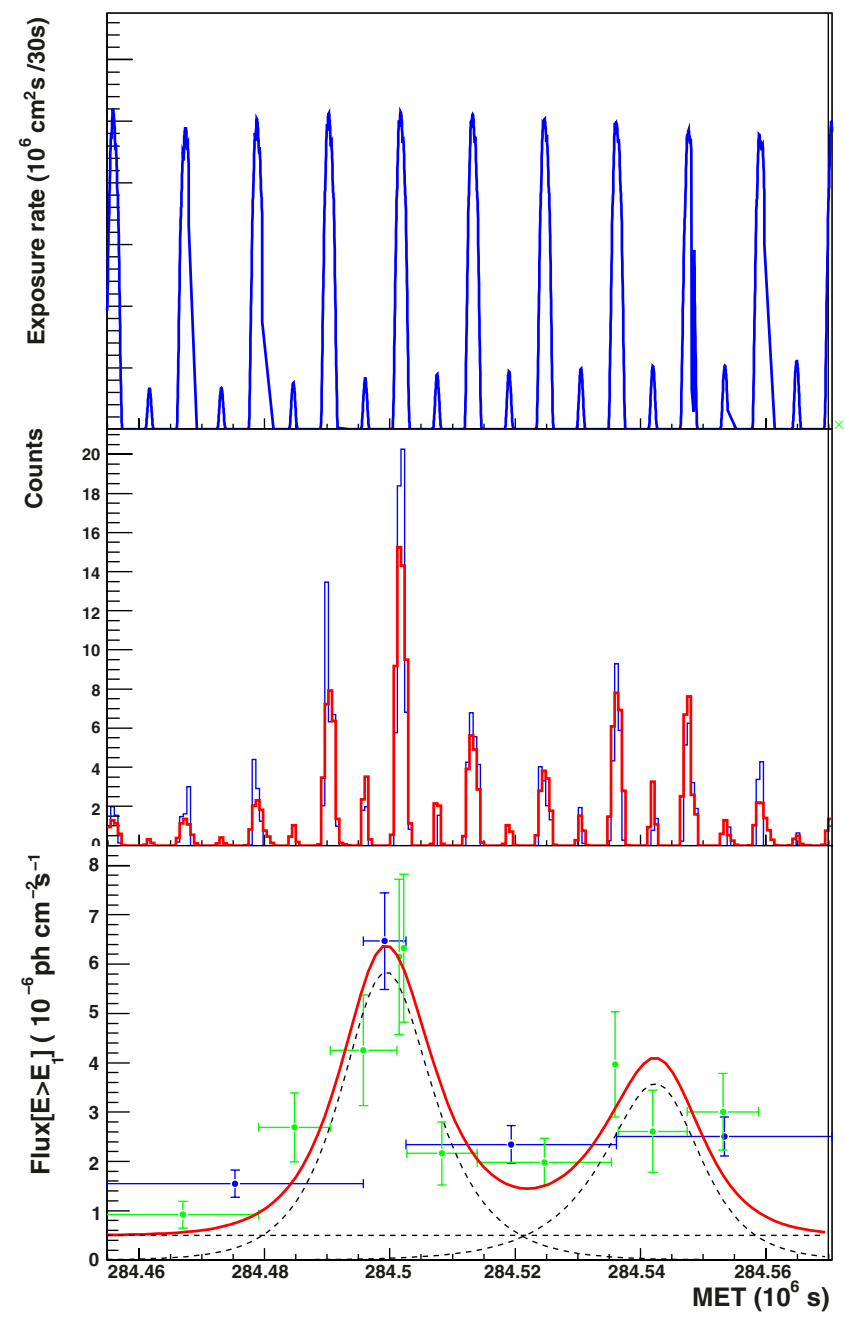

Fig. 3. Top: exposure rate during flare D. Middle: predicted (red) and observed (blue) source photon detection rates. Bottom: photon flux light curve of flare D. The red curve shows the results of the time-domainunbinned analysis, and the blue (15\%) and green (25\%) symbols represent the adaptive-binning analysis results.

activity. As we see in Fig. 6, for each flare $\Gamma$ drops from a value of $\sim(2.9-2.7)$ at the beginning of the flare to $\sim(2.3-2.1)$ close to the peak of the flare, and then at the end of the flare the spectrum softens to a photon index value $\sim(3.0-2.8)$. Therefore, a strong evolution of $\Gamma$ during the different activity states is observed in the source. 


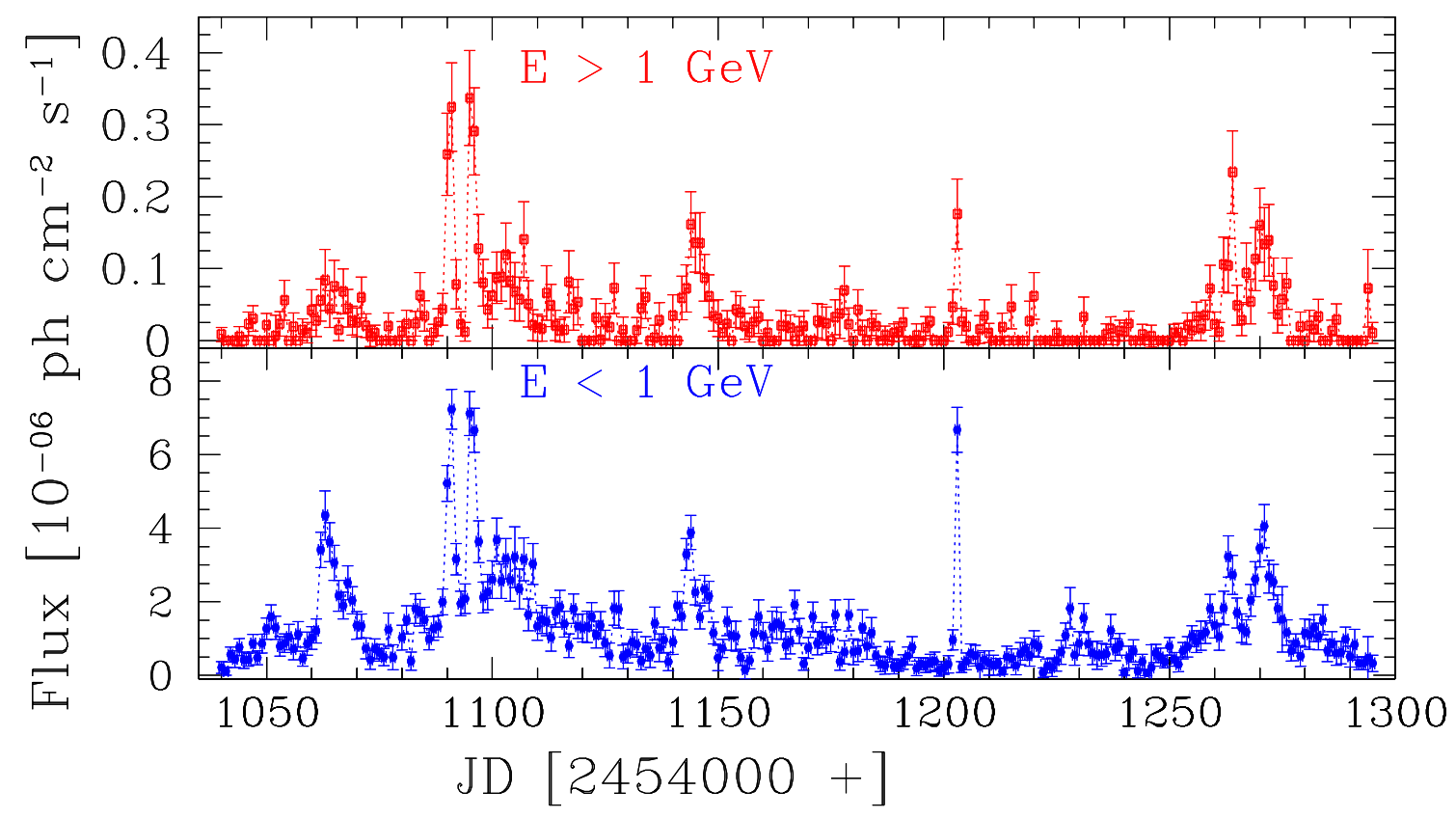

Fig. 4. One-day averaged $\gamma$-ray light curve of the source below (bottom) and above (top) $1 \mathrm{GeV}$ during the high-activity period.

Table 2. Fitted parameters of the bright flares at different energy scales.

\begin{tabular}{lcccccc}
\hline \hline Flare & Sub-flare & $\begin{array}{c}\text { Energy } \\
(\mathrm{GeV})\end{array}$ & $\begin{array}{c}T_{\mathrm{r}} \\
(\text { day })\end{array}$ & $\begin{array}{c}T_{\mathrm{f}} \\
(\text { day) }\end{array}$ & $\begin{array}{c}t_{0} \\
\mathrm{JD}^{\prime}\end{array}$ & $\begin{array}{c}F_{0} \\
10^{-6} \mathrm{ph} \mathrm{cm}^{-2} \mathrm{~s}^{-1}\end{array}$ \\
\hline B & 1 & $0.1-1$ & $0.76 \pm 0.09$ & $1.22 \pm 0.14$ & $1090.92 \pm 0.33$ & $7.15 \pm 0.90$ \\
& & $1-100$ & $0.53 \pm 0.16$ & $1.39 \pm 0.18$ & $1090.81 \pm 0.21$ & $0.35 \pm 0.12$ \\
\hline B & 2 & $0.1-1$ & $0.68 \pm 0.14$ & $1.53 \pm 0.21$ & $1095.02 \pm 0.18$ & $7.21 \pm 0.78$ \\
& & $1-100$ & $0.46 \pm 0.17$ & $1.42 \pm 0.33$ & $1094.93 \pm 0.19$ & $0.30 \pm 0.05$ \\
\hline
\end{tabular}

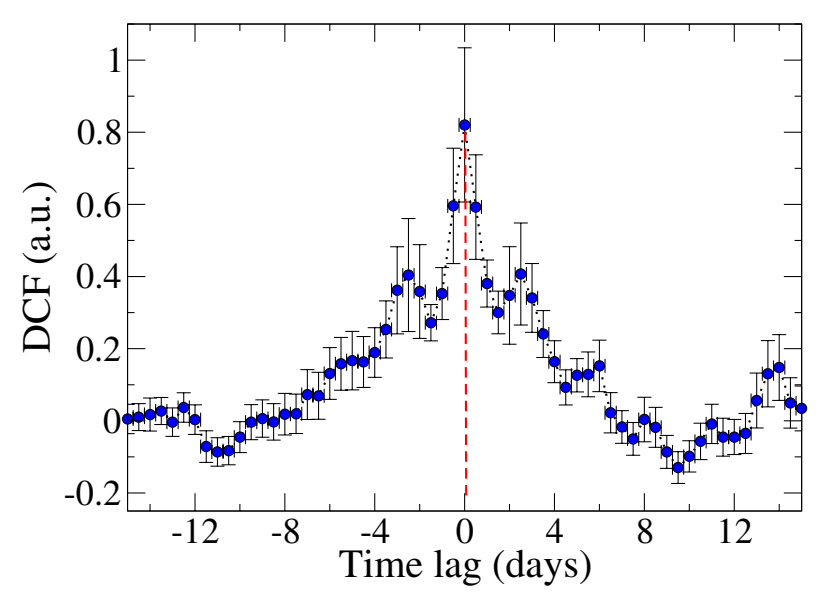

Fig. 5. DCF curve of the $12 \mathrm{~h}$ binned $\gamma$-ray light curves below and above $1 \mathrm{GeV}$. The dotted line marks a possible time lag of $0 \pm 0.25$ day.

An alternative way to investigate the spectral evolution during different $\gamma$-ray flares is to plot variations in $\Gamma$ as a function of flux variations. A counter-clockwise loop is observed during flare $\mathrm{A}$ while during flare $\mathrm{B}$, the spectral index, and flux changes follow a clockwise path (Abdo et al. 2010e). In Fig. 6 (bottom), we also plot the photon indices as a function of the $\gamma$-ray fluxes during flares C, D, and E. As shown, flares C and E suggest a clockwise loop, while flare D follows a counterclockwise loop. A clockwise loop indicates that the flux started to increase at low energy and then propagates to high energy; the reverse would produce a counter-clockwise path. This may reflect the alternating dominance of acceleration and cooling processes. We note that the subsequent flares loop in opposite directions, starting with a counter-clockwise loop for flare A.

\subsection{The gamma-ray spectrum}

We constructed the $\gamma$-ray spectrum using data for the entire flaring period $\left(\mathrm{JD}^{\prime}=1042-1294\right)$ and for the quiescent state $\left(\mathrm{JD}^{\prime}=1300-1720\right)$. Figure 7 a shows the $\gamma$-ray spectrum of the source over the high-activity period and the quiescent state. The observed spectrum shows a clear deviation from a simple power law. The spectral analysis using a broken power law model returns a break energy of $1.5 \pm 0.1 \mathrm{GeV}$ with a likelihood test statistic relative to the power law LRT $=-2 \Delta(\ln L)=35.1$ ( $L$ is the value of the likelihood function) that corresponds to a significance of $\sim 5.58 \sigma$. The significance is estimated using Wilks' theorem, i.e., the LRT follows a chi-square distribution with $\Delta n$ degrees of freedom ( $\Delta n$ is difference of number of degrees of freedom of the two spectral models). However, the spectrum does not exhibit a sharp break; rather, the spectrum shows curvature near the break frequency. We therefore added a smoothing parameter $b$ to the broken power law model (see Sect. 2), which is fixed to $0.1^{4}$ while fitting.

We also investigated the spectral shape using the logparabola $(\log \mathrm{P})$ model. For the high-activity period, the LRT value of SBPL with respect to $\log \mathrm{P}$ is 5.3 , which corresponds to a significance of $\sim 2.3 \sigma$ with one degree of freedom. This implies that the SBPL model provides a marginally better fit

4 The results are not very sensitive to the choice of $b$ values. 

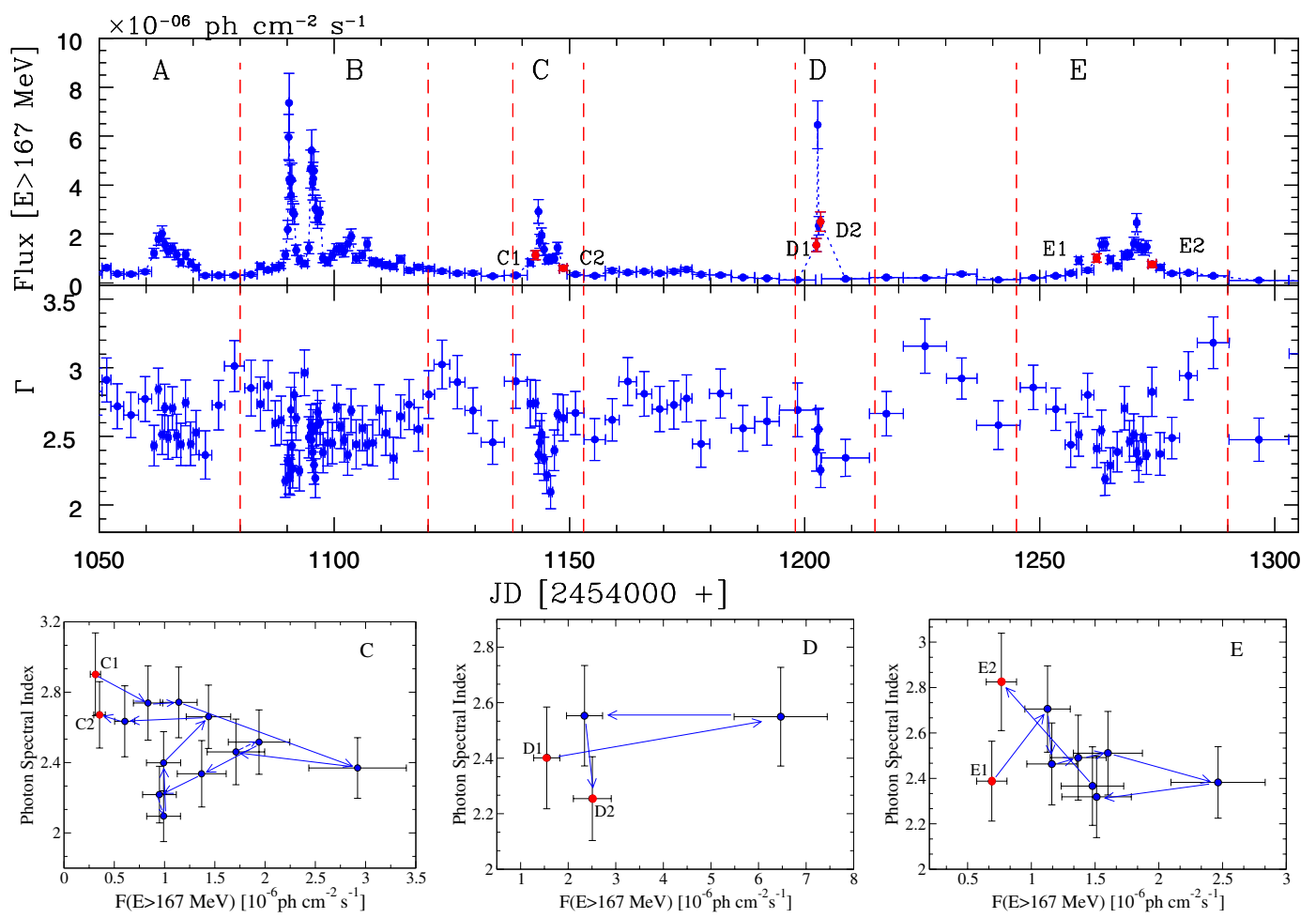

Fig. 6. Top panel: the constant uncertainty (15\%) light curve of 3C 273 above $E_{0}$ obtained with the adaptive binning method over the high-activity period (see text for details). The lower panel shows the variations in $\Gamma$ during this period. The vertical columns separate the different flaring periods. Bottom: $\Gamma$ vs. flux above $E_{0}$ for flare C, D, and E.

compared to the $\log \mathrm{P}$ model. The curves in Fig. 7a represent the fitted power laws, i.e., PL, logP, and SBPL in an energy range between $100 \mathrm{MeV}$ and $300 \mathrm{GeV}$ using the maximum likelihood method. The fitted model parameters over the energy range between $100 \mathrm{MeV}$ to $300 \mathrm{GeV}$ are summarized in Table 3. The difference of the logarithm of likelihood $-2 \Delta L$ of SBPL and $\log P$ with respect to SPL is given in Col. 9 of Table 3 along with the corresponding significance by which SBPL and $\log \mathrm{P}$ are favored compared to the PL model. We therefore conclude that both smoothed broken power law and log-parabola models better describe the $\gamma$-ray spectral shape than the simple PL model with a break energy $E_{\text {break }}=1.2 \pm 0.2 \mathrm{GeV}$. A similar indication of break at $\sim 1.5 \mathrm{GeV}$ in the spectrum of 3C 273 integrated over the first six months of LAT observations has been also reported in Abdo et al. (2010d).

The high-activity period $\left(\mathrm{JD}^{\prime}=1042-1294\right)$ consists of five rapid flares $(\mathrm{A}, \mathrm{B}, \mathrm{C}, \mathrm{D}, \mathrm{E})$. It is very likely that the physical conditions within the emission region change during different flares. We therefore investigated the $\gamma$-ray spectra for the individual flares. Figure 7(b)-(f) show the $\gamma$-ray spectra of the source for the individual flares. For each flare we found that the spectrum shows a smooth rather than sharp break between 1 to $2 \mathrm{GeV}$. As discussed in Sect. 3.1.1, the bright flares (A to E) are further composed of a number of sub-flares. Because of low photon statistics, the curvature in the spectra of the sub-flares cannot be studied. Similarly, the absence of curvature was also noticed by Abdo et al. (2010e) during the two bright $\gamma$-ray sub-flares (subcomponents of Flare B).

The variation of break energy (calculated using SBPL) with the photon flux variations is displayed in Fig. 8a. As we see here, no strong variation in break energy with respect to the flux variation is found in the source. This is similar to other bright Fermi blazars (Abdo et al. 2011; Ackermann et al. 2010; Rani et al. 2013b). The break energy $E_{\text {break }}$ varies within a factor of 2 , while, the flux varies by a factor of $\approx 16$. Figure $8 \mathrm{~b}$ displays the variation of $\Delta \Gamma=\Gamma_{2}-\Gamma_{1}$ (see Sect. 2) with the break energy $\left(E_{\text {break }}\right)$. Again, the break energy remains nearly constant for a significant variation in $\Delta \Gamma$.

\subsection{Gamma-ray Doppler factor}

The minimum Doppler factor $\delta_{\gamma}$ can be evaluated using the fact that the high-energy $\gamma$-ray photons can collide with the softer radiation to produce $e^{ \pm}$pairs. The cross-section of this process is maximized $\sim \sigma_{\mathrm{T}} / 5$ (Svensson 1987), where $\sigma_{\mathrm{T}}$ is the Thompson scattering cross-section. This leads to a lower limit on $\delta$ with the requirement that the optical depth $\tau_{\gamma \gamma}(v)<1$ (Dondi \& Ghisellini 1995; Finke et al. 2008),

$\delta_{\gamma}>\left[\frac{2^{a-1}(1+z)^{2-2 a} \sigma_{\mathrm{T}} d_{L}^{2}}{m_{\mathrm{e}} c^{4} t_{\mathrm{var}}} \epsilon f_{\epsilon^{-1}}^{\mathrm{syn}}\right]^{\frac{1}{6-2 a}}$,

where $a$ is the power law index of synchrotron flux, i.e., $f_{\epsilon}^{\text {syn }} \propto$ $\epsilon^{a}, m_{\mathrm{e}}$ is the electron mass, and $\epsilon=E /\left(m_{\mathrm{e}} c^{2}\right)$ is the dimensionless energy of the $\gamma$-ray photon with energy $E$ for which the optical depth of the emitting region $\tau=1$. The luminosity distance $d_{L}$ corresponding to $z=0.158$ (Strauss et al. 1992) is $d_{L}=0.749 \mathrm{Gpc}^{5}$ for a $\Lambda \mathrm{CDM}$ cosmology with $\Omega_{\mathrm{m}}=0.27$, $\Omega_{\lambda}=0.73$, and $\mathrm{H}_{0}=71 \mathrm{~km} \mathrm{~s}^{-1} \mathrm{Mpc}^{-1}$ (Spergel et al. 2003). For the highest energy $\gamma$-ray $(15.4 \mathrm{GeV})$ photon observed in the source, we obtain $\epsilon=15.4 \mathrm{GeV} /\left(5.11 \times 10^{-4} \mathrm{GeV}\right)=3 \times 10^{4}$ and $\epsilon^{-1}=3.3 \times 10^{-5}$. Using $f_{\epsilon^{-1}}^{\text {syn }}=4 \times 10^{-10} \mathrm{erg} \mathrm{cm}^{-2} \mathrm{~s}^{-1}$, observed by Swift/UVOT from January 10 to 13, 2010 (Giommi et al. 2012) and $t_{\mathrm{var}}=0.07$ days (taken as the rising time of

5 The results are not sensitive to $d_{L}=0.784 \mathrm{Gpc}$ for the recent Planck measurements of cosmology parameters (Planck Collaboration et al. 2013). 
B. Rani et al.: $\mathrm{GeV}$ variability in $3 \mathrm{C} 273$
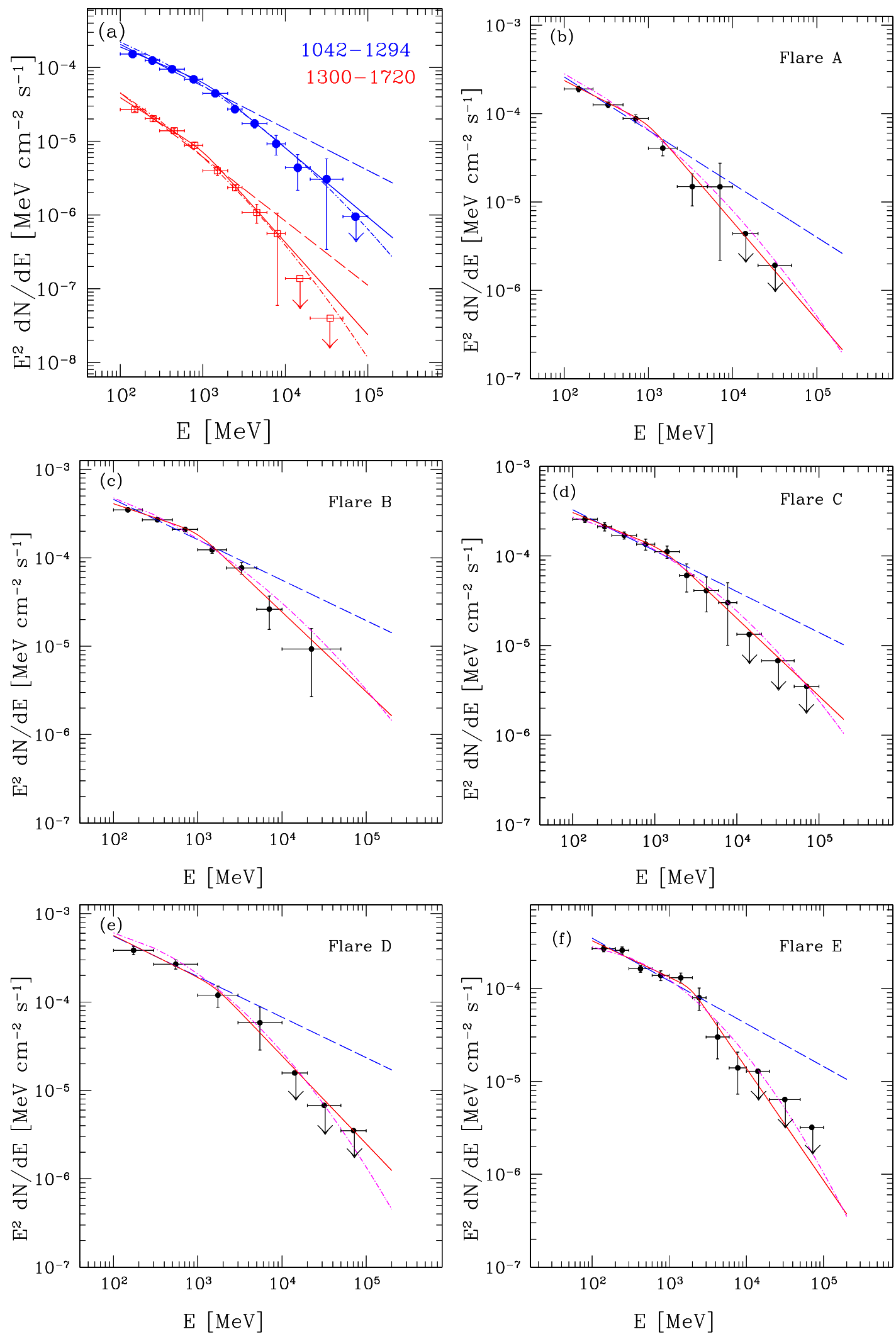

Fig. 7. Gamma-ray spectral energy distributions of 3C 273 during different activity states along with the fitted PL (dashed curve), logP (dotteddashed curve), and SBPL (solid curve) spectral models.

the most rapid flare D), we obtain $\delta_{\gamma} \geq 10.4$. The estimated $\delta_{\gamma}$ value is comparable to $\delta_{\text {VLBI }}=5-12$ found by Savolainen et al. (2006) and Jorstad et al. (2005) using kinematics of the parsec scale VLBI jet derived at radio wavelength.

\subsection{Size of the emission region}

One can also obtain an estimate of the size of the emission region $(R)$ using the the calculated Doppler factor and variability time 
Table 3. Parameters of fitted power laws.

\begin{tabular}{|c|c|c|c|c|c|c|c|c|c|}
\hline Bin & $\begin{array}{c}\mathrm{JD}^{\prime} \\
{[\mathrm{JD}-2454 \text { 000] }}\end{array}$ & $\begin{array}{c}F_{100} \\
\left(10^{-6} \mathrm{ph} \mathrm{cm}^{-2} \mathrm{~s}^{-1}\right)\end{array}$ & Model & $\Gamma / \alpha / \Gamma_{1}$ & $\beta / \Gamma_{2}$ & $\begin{array}{c}E_{\text {break }} \\
(\mathrm{GeV})\end{array}$ & $\begin{array}{c}\Delta \Gamma \\
\left(\Gamma_{2}-\Gamma_{1}\right)\end{array}$ & $-2 \Delta(\ln L)$ & Significance \\
\hline \multirow{3}{*}{ high } & $1042-1294$ & $1.31 \pm 0.02$ & PL & $2.57 \pm 0.01$ & & - & & & \\
\hline & & & $\log \mathrm{P}$ & $2.74 \pm 0.03$ & $0.08 \pm 0.01$ & $\ldots$ & & 44.7 & $6.7 \sigma$ \\
\hline & & & SBPL & $2.47 \pm 0.02$ & $2.94 \pm 0.06$ & $1.2 \pm 0.2$ & $0.47 \pm 0.06$ & 40.5 & $6.1 \sigma$ \\
\hline \multirow[t]{3}{*}{ low } & $1300-1720$ & $0.24 \pm 0.10$ & PL & $2.87 \pm 0.05$ & & $\ldots$ & & & \\
\hline & & & $\log \mathrm{P}$ & $2.86 \pm 0.04$ & $0.09 \pm 0.04$ & $\ldots$ & & 9.9 & $3.3 \sigma$ \\
\hline & & & SBPL & $2.70 \pm 0.01$ & $3.25 \pm 0.10$ & $1.0 \pm 0.2$ & $0.55 \pm 0.10$ & 11.9 & $3.3 \sigma$ \\
\hline \multirow[t]{3}{*}{ Flare A } & $1058-1078$ & $1.62 \pm 0.07$ & PL & $2.60 \pm 0.05$ & & $\ldots$ & & & \\
\hline & & & $\log \mathrm{P}$ & $2.82 \pm 0.10$ & $0.11 \pm 0.04$ & & & 6.3 & $2.6 \sigma$ \\
\hline & & & SBPL & $2.48 \pm 0.08$ & $3.11 \pm 0.28$ & $1.0 \pm 0.2$ & $0.63 \pm 0.28$ & 9.8 & $2.9 \sigma$ \\
\hline \multirow[t]{3}{*}{ Flare B } & $1086-1114$ & $3.15 \pm 0.08$ & PL & $2.45 \pm 0.03$ & & $\ldots$ & & & \\
\hline & & & $\log \mathrm{P}$ & $2.63 \pm 0.13$ & $0.12 \pm 0.06$ & $\ldots$ & & 29.6 & $5.1 \sigma$ \\
\hline & & & SBPL & $2.32 \pm 0.02$ & $2.90 \pm 0.09$ & $1.2 \pm 0.2$ & $0.58 \pm 0.09$ & 24.9 & $4.7 \sigma$ \\
\hline \multirow[t]{3}{*}{ Flare C } & $1140-1150$ & $2.25 \pm 0.11$ & PL & $2.45 \pm 0.04$ & & $\ldots$ & & & \\
\hline & & & $\log \mathrm{P}$ & $2.56 \pm 0.09$ & $0.06 \pm 0.02$ & $\ldots$ & & 7.3 & $2.9 \sigma$ \\
\hline & & & SBPL & $2.37 \pm 0.03$ & $2.86 \pm 0.08$ & $1.3 \pm 0.2$ & $0.49 \pm 0.08$ & 9.9 & $2.9 \sigma$ \\
\hline \multirow[t]{3}{*}{ Flare D } & $1202-1204$ & $3.83 \pm 0.32$ & PL & $2.46 \pm 0.08$ & & $\ldots$ & & & \\
\hline & & & $\log \mathrm{P}$ & $2.74 \pm 0.12$ & $0.25 \pm 0.06$ & $\ldots$ & & 9.8 & $3.3 \sigma$ \\
\hline & & & SBPL & $2.47 \pm 0.10$ & $2.99 \pm 0.13$ & $1.6 \pm 0.2$ & $0.52 \pm 0.16$ & 6.3 & $2.2 \sigma$ \\
\hline \multirow{3}{*}{ Flare E } & $1260-1275$ & $2.38 \pm 0.11$ & PL & $2.46 \pm 0.02$ & & $\ldots$ & & & \\
\hline & & & $\log \mathrm{P}$ & $2.63 \pm 0.18$ & $0.09 \pm 0.07$ & $\ldots$ & & 7.2 & $2.7 \sigma$ \\
\hline & & & SBPL & $2.36 \pm 0.05$ & $3.72 \pm 0.18$ & $1.9 \pm 0.2$ & $1.36 \pm 0.18$ & 12.1 & $3.1 \sigma$ \\
\hline
\end{tabular}

Notes. $\Delta(\ln L)$ is the difference of the $-\log \left(\right.$ likelihood) value of $\log$, SBPL with respect to PL. ${ }^{(a)}$ We follow Wilks' theorem to estimate the significance, i.e., twice the difference between the $\log \left(\right.$ likelihood) values for the two spectral models is formally distributed as $\chi^{2}$ with $\Delta n$ degrees of freedom, where $\Delta n$ is the difference in the number of degrees of freedom between the two models.
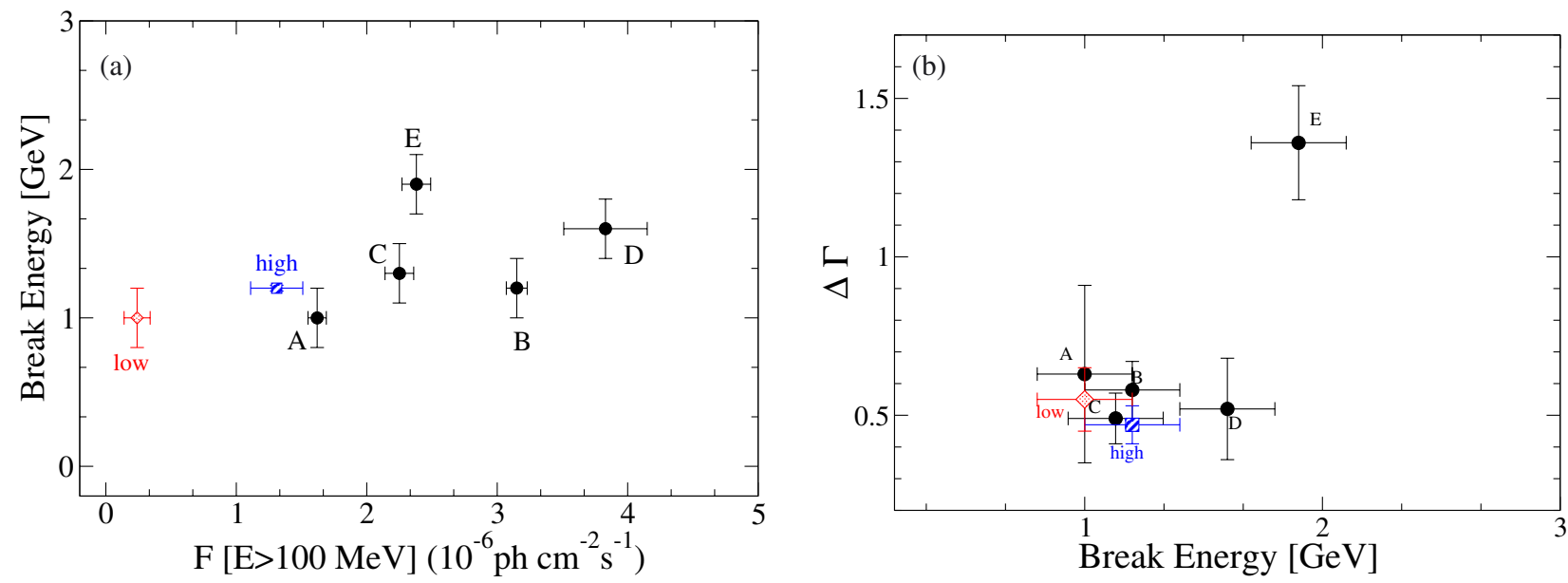

Fig. 8. a) Break energy plotted as a function of flux for the different activity periods. b) Change of the spectral slope $\Delta \Gamma$ as a function of break energy. In both plots, the squares represent the high-activity period, the diamonds the low-activity period, and the circles are the individual flares during the high-activity period.

scale ( $\left.t_{\mathrm{var}}\right)$, i.e., $R \leq c t_{\mathrm{var}} \delta_{\gamma} /(1+z)$ (Rani et al. 2013a). Using $t_{\text {var }}=0.07$ days, we obtain $R \leq 1.6 \times 10^{14} \delta_{\gamma} \mathrm{cm}$. A lower limit of the estimated Doppler factor $\delta_{\gamma} \geq 10.4$ gives $R \cong 1.6 \times 10^{15} \mathrm{~cm}$.

The angular size of the emission region is calculated using the equation (Rani et al. 2013a)

$\theta \leq 0.173 \frac{t_{\mathrm{var}}}{d_{\mathrm{L}}} \delta_{\gamma}(1+\mathrm{z}) \mathrm{mas}$,

where $d_{L}$ is is the luminosity distance in Gpc. Using $t_{\mathrm{var}}=$ 0.07 days, $d_{L}=0.749 \mathrm{Gpc}, \delta_{\gamma} \geq 10.4$, we obtain $\theta \cong 0.52 \mu$ as which is much smaller than the size of the core region $\theta_{\text {core }}=$ $(70 \pm 10) \mu$ as estimated using VLBA observations (Savolainen et al. 2008). It is worth pointing out that the $R$ and $\theta$ values found in this section could be smaller, since the variability timescale only gives upper limits, or larger, since the Doppler factor used is a lower limit.

\section{Discussion}

\subsection{Rapid gamma-ray flares}

A prominent flaring activity was observed in the source between August 2009 and April 2010. The high-activity period of the source was later followed by a quiescent state which is still continuing. The fastest $\gamma$-ray flare observed in the source had a flux doubling timescale of $1.1 \mathrm{~h}$. Gamma-ray flares on similar time scales are also observed in many other bright Fermiblazars (Abdo et al. 2010c; Foschini et al. 2011, 2013; Saito et al. 2013; Nalewajko 2013; Abdo et al. 2011). Flux doubling times in the GeV band have been reported in PKS1510-089 to be less than 3 h (Brown 2013; Saito et al. 2013) or as short as $20 \mathrm{~min}$ (Foschini et al. 2013). Although these analyses did not properly take into account the discontinuous LAT exposure pattern as our method did, we conclude that the fastest flare observed in 3C 273 
is comparable to the most rapid flares seen in other Fermi-LAT detected blazars.

Compared to the asymmetric profile of the very strong events of September 2009 (flare B), flares A, C, D, and E are characterized by symmetric profiles. The asymmetric profile can be explored in terms of a fast injection of accelerated particles and a slower radiative cooling and/or escape from the active region (Sikora et al. 2001). The result of the superposition and blending of several episodes of short duration could provide symmetric flare shapes (Valtaoja et al. 1999). Although the authors only consider external Comptonization in their model, it is worth pointing out that synchrotron self-Comptonization is also possible. Nalewajko (2013) argued that different Doppler boosting for different shells of the emission region could also be responsible for the asymmetry of $\gamma$-ray flares.

\subsection{Origin of spectral breaks}

Our analysis shows that the $\gamma$-ray spectrum of the source clearly deviates from a simple power law and is better described by a smooth break at $\sim 1.2 \mathrm{GeV}$. For the individual bright flares the break energy lies between 1-2 GeV. During the individual flares, the change in spectral slope above and below the break energy, $\Delta \Gamma$, varies between 0.47 to 1.36 .

The observed spectral breaks in many bright Fermi blazars mostly lie within 2-10 GeVs (Poutanen \& Stern 2010; Tanaka et al. 2011). Among the proposed scenarios, the absorption of $\gamma$-rays via photon-photon pair production on He II Lyman recombination continuum and lines within the broad-line region (BLR; e.g., Poutanen \& Stern 2010; Tanaka et al. 2011, and references therein) can be responsible for the observed breaks. The $\gamma$-ray emitting region must be located within the BLR for this model to work. Alternatively, the $\gamma$-ray spectral breaks could also be explained by a combination of two Compton-scattered components, i.e., Compton scattering of the disk and BLR radiation as proposed by Finke \& Dermer (2010). Dermer et al. (in prep.) proposes a three-parameter log-parabola model for the electron energy distribution, which naturally produces a $\mathrm{GeV}$ spectral cutoff by scattering Ly- $\alpha$ and BLR radiation. Another explanation refers to an intrinsic origin of the spectral breaks. A change in spectral index below and above the break of order $\sim 0.5$ can be interpreted as a typical cooling break associated with radiative losses (Abdo et al. 2009). The observed softening may instead be due to an intrinsic decline or break in the particle distribution also.

For 3C 273, the change in spectral slope $(\Delta \Gamma)$ above and below the break energy varies between 0.47 to 1.36 . The estimated $\Delta \Gamma$ values for flare $\mathrm{E}$ with $\Delta \Gamma \sim 1.36$ do not favor the standard radiative cooling models that predict a spectral break of 0.5 units. Although for flare $\mathrm{A}$ to $\mathrm{D}, \Delta \Gamma$ is close to 0.5 , but it is difficult to reconcile the constancy of the break energy with respect to the photon flux variations within the cooling break scenario. This does not argue in favor of the intrinsic origin of the observed spectral breaks in 3C 273 associated with the radiative cooling.

If the spectral breaks are due to $\gamma \gamma$ absorption, then one can estimate the frequency of the most efficiently interacting target photons with $\gamma$-rays. For head-on collisions, we have $v \approx 6 \times 10^{14}(E / 100 \mathrm{GeV})^{-1} \mathrm{~Hz}$. A spectral break at $E_{\text {break }}=$ $1-2 \mathrm{GeV}$ in the observer frame corresponds to $0.86-1.73 \mathrm{GeV}$ $\left(E_{\text {break }} /(1+z)\right)$ in the source frame. Therefore, for the spectral break within $0.86-1.73 \mathrm{GeV}$, the target photons fall within (3.4-6.9) $\times 10^{16} \mathrm{~Hz}$, i.e., are extreme ultra-violet photons. This implies that the target photon energy is higher than the He II and $\mathrm{H}$ I recombination continuum. Moreover, spectral breaks close to
$1 \mathrm{GeV}$ are not very likely to be observed within this scenario because of low opacity at these energies (Poutanen \& Stern 2010).

The optical-UV spectrum of the FSRQ 3C 273 shows a prominent excess of emission, which is mainly interpreted as a contribution of the accretion disk emission (see Ulrich 1981; Soldi et al. 2008, and references therein) or the presence of a hot corona particularly in the UV-Xray regime (Haardt et al. 1994). The excess optical-UV emission can be approximated by a black body with a temperature of $\sim 21000-26000 \mathrm{~K}$ with a characteristic dimension of $10^{16} \mathrm{~cm}$ (Ulrich 1981; Pian et al. 1999). Consequently, Compton processes of a thermal plasma in the disk or in the corona can contribute efficiently to the highenergy radiation. The optical-UV spectrum of 3C 273 also shows strong emission lines, i.e., Ly- $\alpha$, CIV, OVI, CIII, NIII, and SVI (e.g., Appenzeller et al. 1998; Paltani \& Türler 2003, and references therein). Therefore it might not be surprising to find a contribution of inverse-Compton scattered radiation from both the disk and the BLR.

\subsection{Location of the gamma-ray emission region}

Assuming a conical geometry, the observed variability timescale of the source can also be used to put an upper limit on the distance of the emission region $\left(r_{\gamma}\right)$ from the central engine (Tavecchio et al. 2010) using $r_{\gamma}<\left(t_{\mathrm{var}} \mathrm{c} \delta\right) /\left(\theta_{\text {jet }}(1+z)\right)$, where $\theta_{\text {jet }}$ is the opening angle of the jet. Moreover, as we are using a lower limit of Doppler factor $\delta_{\gamma}$, the estimated value of $r_{\gamma}$ is supposed to be close to the actual value. Using the rising time of flare D, $t_{\mathrm{var}}=0.07$ day, $\delta_{\gamma}=10$, and $\theta_{\text {jet }} \sim 1.4^{\circ}$ (Jorstad et al. 2005), we obtain $r_{\gamma} \sim 0.03$ pc. However we stress that this is a very rough assumption as the jet may not be conical (Hada et al. 2012; Krichbaum et al. 2006). Moreover, the $\gamma$-ray emitting region of the jet could be much closer to the black hole (with a different Doppler factor) compared to the portion of the jet resolved by the VLBI (Rani et al. 2013a).

The energy dependence of the cooling timescale can also be used as an alternative approach to constrain the location of the $\gamma$-ray emission region (Dotson et al. 2012). For 3C 273, we found that the cooling timescales of the bright $\gamma$-ray flares are similar for $E<1 \mathrm{GeV}$ and $E>1 \mathrm{GeV}$ light curves with a possible minimum temporal resolution of $6 \mathrm{~h}$. This implies that the allowed difference for $F_{E<1 \mathrm{GeV}}$ and $F_{E>1 \mathrm{GeV}}$ decay timescale is shorter than $6 \mathrm{~h}$. An upper limit for the distance of the $\gamma$-ray emission region from the central engine is given by $r_{\gamma}<2.3 \times 10^{19} \Gamma_{\text {bulk }}\left[\Delta t_{\text {max,hr }} L_{\mathrm{MT}, 45} /(1+z)^{1 / 2}\right]^{1 / 2} \mathrm{~cm}$, where $L_{\mathrm{MT}, 45}$ is the molecular torus luminosity in units of $10^{45} \mathrm{erg} \mathrm{s}^{-1}$ and $\Gamma_{\text {bulk }}$ is the bulk Lorentz factor (Dotson et al. 2012). The molecular torus luminosity can be considered as a $0.1-0.5$ fraction of the accretion disk luminosity $\left(L_{\mathrm{AD}}\right)$. For $3 \mathrm{C} 273$, we have $L_{\mathrm{AD}} \sim 1.6 \times 10^{46} \mathrm{erg} \mathrm{s}^{-1}$ (Kriss et al. 1999; Soldi et al. 2008; Giommi et al. 2012). Using $L_{\mathrm{MT}, 45} \sim 1.6, \Gamma_{\text {bulk,10 }}=10.6 \pm 2.8$ (Jorstad et al. 2005) and $\Delta t_{\max }<6 \mathrm{~h}$, we obtain $r_{\gamma}<1.6 \mathrm{pc}$.

Finke \& Dermer (2010) argue that a region where the Compton-scattered disk and BLR emissions are approximately equal can explain the Fermi-LAT emission in 3C 454.3. Their analysis implies that the location of $\gamma$-ray emission region $\left(r_{\gamma}\right)$ is $R_{i}<r_{\gamma} \ll \Gamma_{\text {bulk }}^{4} r_{g}$, where $R_{i}$ is the inner radius of BLR, $\Gamma_{\text {bulk }}$ is the bulk Lorentz factor, and $r_{g}$ is Schwarzschild radius of the central black hole. They also propose a robust solution where the external field energy density from disk and BLR radiation decays as $\sim r^{-3}$. In this scenario, a comparison of BLR and disk radiation gives $\tau_{\mathrm{BLR}}=\left(R_{i} / r_{g}\right)^{-1}\left(\tau_{\mathrm{BLR}}\right.$ is the Thomson depth of the BLR) for the emission regions formed in the BLR. 
For 3C $273, M_{9}=M_{\mathrm{BH}} /\left(10^{9} M_{\odot}\right) \approx 0.9-2.4$ (Paltani \& Türler 2005; Peterson et al. 2004), where $M_{\mathrm{BH}}$ is the black hole mass. Using $\tau_{\mathrm{BLR}}=0.01$ to 0.1 (Boettcher \& Dermer 1998), and $\Gamma_{\text {bulk }}=10.6 \pm 2.8($ Jorstad et al. 2005), we obtain $0.005 \mathrm{pc}<$ $r_{\gamma}<1.4 \mathrm{pc}$. The achromatic cooling of flares constrains $r_{\gamma}$ within $1.6 \mathrm{pc}$. Therefore, the allowed range of $r_{\gamma}$ using the two methods is $0.005 \mathrm{pc}<r_{\gamma}<1.6 \mathrm{pc}$.

Our analysis suggests that the location of the $\gamma$-ray flaring activity observed in 3C 273 lies within $1.6 \mathrm{pc}$ distance from the central engine. The achromatic cooling of the $\gamma$-ray flares below and above $1 \mathrm{GeV}$ is consistent with the $\gamma$-ray emission region being located within the BLR. Also, the observed spectral breaks (at $\sim 1-2 \mathrm{GeV}$ ) can be well described by $\gamma$-ray absorption within the BLR. Moreover, as a result of $\gamma \gamma$ pair production, the BLR of FSRQs is opaque to $\gamma$-rays above $\sim 20 \mathrm{GeV}$, while the MT region is not (Donea \& Protheroe 2003). As we saw in Sect. 3.1, the highest energy $\gamma$-ray photon observed for 3C 273 has energy of $15.4 \mathrm{GeV}$, and provided the fact that the source has not been detected at $\mathrm{TeV}$ energies so far; therefore, our results are consistent with the $\gamma$-ray emission site being located within the BLR.

\subsection{The orphan flare $E$}

We found that the $\Delta \Gamma$ for flare $E(\sim 1.5)$ is significantly different from that for flares A to $\mathrm{D}(\sim 0.5)$. We note that flare $\mathrm{E}$ is the last flare observed during the high-activity period. Also, the break energy of flare $\mathrm{E}\left(E_{\mathrm{break}} \sim 2 \mathrm{GeV}\right)$ is higher than for the rest of the flares. Moreover, the doubling time scales of flare E are comparatively longer than those for flares A to D. We also notice that in the monthly averaged light curves (Fig. 1), flares A to D are the sub-components of a single flaring event, while flare $\mathrm{E}$ seems to be an independent flaring event.

The impact of the geometry of the BLR on the expected absorption, through the $\gamma \gamma$ process was recently discussed by Tavecchio \& Ghisellini (2012). They argued that if the BLR has a full covering factor, the break energy does not change as long as the emission occurs within the BLR, but $\Delta \Gamma$ decreases as the emission region moves away from the central engine. A correlated $\Delta \Gamma-E_{\text {break }}$ variation is expected for the partially covered BLR. For flare A to $D$, the nearly constant values of $\Delta \Gamma$ and $E_{\text {break }}$ suggest an approximately fixed location of the emission region from the black hole. The higher $\Delta \Gamma$ value for flare $\mathrm{E}$ implies a closer location to the central engine compared to flare A to D. However, the longer variability timescale for flare E could argue for an emitting region larger and farther out (if the jet is not stratified), contrary to the result from the larger $\Delta \Gamma$.

With the recent jet kinematics study of 3C 273 at $7 \mathrm{~mm}$ wavelength, Jorstad et al. (2012) reported an ejection of four components during the high $\gamma$-ray activity period between mid2009 to mid-2010. The authors identified two components during the high $\gamma$-ray activity period. The ejection of the fastest knot was associated with the most prominent $\gamma$-ray peak (flare B), while the brightest component had maximum flux coinciding with flare E. This suggests that the flares A-D and E are independent flaring events. Multiple shock scenarios and/or shockshock interactions are among the feasible mechanisms for these flaring events (Sokolov \& Marscher 2004; Fromm et al. 2012). It could also be possible that flare $\mathrm{E}$ is located at a similar distance from the central engine to flares A-D, but is in a different layer or sheath in the jet. This scenario is expected within the multi-zone emission model of Marscher (2013), which describes a stratified jet.

\section{Conclusions}

The continuous monitoring in the high-energy $\gamma$-ray band by the Fermi-LAT allows us to investigate the $\gamma$-ray flux and spectral variability of the FSRQ 3C 273. The source displays prominent flaring activity from August 2009 to April 2010. Five rapid flares are observed in the source during the interval. The rapid flares recur roughly at 50 day intervals. Each individual flare is also composed of sub-flaring components with characteristic rise and decay timescales as fast as few hours. The fastest $\gamma$-ray flare observed in the source has a characteristic flux doubling time scale of $1.1 \mathrm{~h}$ and is compatible with the fast $\gamma$-ray flares observed in other Fermi blazars. The $3 \mathrm{~h}$ peak flux of the flare above $100 \mathrm{MeV}$ is $(12.3 \pm 2) \times 10^{-6} \mathrm{ph} \mathrm{cm}^{-2} \mathrm{~s}^{-1}$, corresponding to an apparent isotropic $\gamma$-ray luminosity of $2.6 \times 10^{46} \mathrm{erg} \mathrm{s}^{-1}$. The source exhibits a strong evolution of spectral index $(\Gamma)$ during the different activity states.

The highest energy $\gamma$-ray photon $(15.4 \mathrm{GeV})$ observed for the source arrives during this high-activity period. The minimum $\gamma$-ray Doppler factor $\delta_{\gamma}$ derived using $\gamma \gamma$-opacity constraints for the highest energy $\gamma$-ray photon is 10 , which is comparable to $\delta_{\text {VLBI }} \sim 5-11$ obtained using jet kinematics (Savolainen et al. 2006; Jorstad et al. 2005). Causality arguments constrain the size of the emission region to $1.6 \times 10^{15} \mathrm{~cm}$.

The $\gamma$-ray spectra measured over this period show clear deviation from a simple power law with a break in the $1-2 \mathrm{GeV}$ energy range. During different flaring epochs, no strong evolution of $E_{\text {break }}$ is found. We have discussed possible explanations for the origin of $\gamma$-ray spectral breaks. The different possible scenarios allow us to constrain the location of $\gamma$-ray emission region(s) within the BLR $(<1.6 \mathrm{pc})$. We argue that the observed $\gamma$-ray emission in 3C 273 is unlikely to have a contribution of the molecular torus photons in external Comptonization. We found that the spectral parameters $\left(E_{\text {break }}\right.$ and $\left.\Delta \Gamma\right)$ of flare $E$ are not comparable to the spectral parameters of flares A to D. A comparison of the $\gamma$-ray flares with the jet kinematics (Jorstad et al. 2012) suggests that flare $E$ has a different physical origin. A detailed discussion of physical mechanisms, along with results of the study focused on broadband flux variability and spectral modeling of the source will shed more light on this, and will be given in a separate paper (Rani et al., in prep.).

Acknowledgements. The Fermi-LAT Collaboration acknowledges generous ongoing support from a number of agencies and institutes that have supported both the development and the operation of the LAT as well as scientific data analysis. These include the National Aeronautics and Space Administration and the Department of Energy in the United States, the Commissariat á l'Énergie Atomique and the Centre National de la Recherche Scientifique/Institut National de Physique Nucléaire et de Physique des Particules in France, the Agenzia Spaziale Italiana and the Istituto Nazionale di Fisica Nucleare in Italy, the Ministry of Education, Culture, Sports, Science and Technology (MEXT), High Energy Accelerator Research Organization (KEK), and Japan Aerospace Exploration Agency (JAXA) in Japan, and the K. A. Wallenberg Foundation, the Swedish Research Council, and the Swedish National Space Board in Sweden. Additional support for science analysis during the operations phase is gratefully acknowledged from the Istituto Nazionale di Astrofisica in Italy and the Centre National d'Études Spatiales in France. We would like to thank Stefano Ciprini, the internal referee for the Fermi-LAT Collaboration, Stefanie Komossa, Seth Digel, Marco Ajello, and Justin Finke for their useful suggestions and comments. We thank the referee for constructive comments that have helped us to improve the paper. B.R. gratefully acknowledges the travel support the COSPAR Capacity-Building Workshop fellowship program.

\section{References}

Abdo, A. A., Ackermann, M., Ajello, M., et al. 2009, ApJS, 183, 46 Abdo, A. A., Ackermann, M., Agudo, I., et al. 2010a, ApJ, 716, 30 Abdo, A. A., Ackermann, M., Ajello, M., et al. 2010b, ApJ, 722, 520 
Abdo, A. A., Ackermann, M., Ajello, M., et al. 2010c, ApJ, 710, 810 Abdo, A. A., Ackermann, M., Ajello, M., et al. 2010d, ApJ, 710, 1271 Abdo, A. A., Ackermann, M., Ajello, M., et al. 2010e, ApJ, 714, L73 Abdo, A. A., Ackermann, M., Ajello, M., et al. 2011, ApJ, 733, L26 Ackermann, M., Ajello, M., Baldini, L., et al. 2010, ApJ, 721, 1383 Ackermann, M., Ajello, M., Albert, A., et al. 2012, ApJS, 203, 4 Appenzeller, I., Krautter, J., Mandel, H., et al. 1998, ApJ, 500, L9 Bastieri, D. 2009, The Astronomer's Telegram, 2168, 1

Boettcher, M., \& Dermer, C. D. 1998, ApJ, 501, L51

Brown, A. M. 2013, MNRAS, 431, 824

Dondi, L., \& Ghisellini, G. 1995, MNRAS, 273, 583

Donea, A.-C., \& Protheroe, R. J. 2003, Astropart. Phys., 18, 377

Dotson, A., Georganopoulos, M., Kazanas, D., \& Perlman, E. S. 2012, ApJ, 758, L15

Edelson, R. A., \& Krolik, J. H. 1988, ApJ, 333, 646

Escande, L., \& Tanaka, Y. T. 2009, The Astronomer's Telegram, 2328, 1

Finke, J. D., \& Dermer, C. D. 2010, ApJ, 714, L303

Finke, J. D., Dermer, C. D., \& Böttcher, M. 2008, ApJ, 686, 181

Foschini, L., Ghisellini, G., Tavecchio, F., Bonnoli, G., \& Stamerra, A. 2011, A\&A, 530, A77

Foschini, L., Bonnoli, G., Ghisellini, G., et al. 2013, A\&A, 555, A138

Fromm, C. M., Perucho, M., Ros, E., et al. 2012, Int. J. Mod. Phys. Conf. Ser., 8,323

Giommi, P., Polenta, G., Lähteenmäki, A., et al. 2012, A\&A, 541, A160

Haardt, F., Maraschi, L., \& Ghisellini, G. 1994, ApJ, 432, L95

Hada, K., Kino, M., Nagai, H., et al. 2012, ApJ, 760, 52

Hartman, R. C., Bertsch, D. L., Bloom, S. D., et al. 1999, ApJS, 123, 79

Jorstad, S. G., Marscher, A. P., Lister, M. L., et al. 2005, AJ, 130, 1418

Jorstad, S., Marscher, A., Smith, P., et al. 2012, Int. J. Mod. Phys. Conf. Ser., 8, 356

Krichbaum, T. P., Graham, D. A., Witzel, A., et al. 2001, in Particles and Fields in Radio Galaxies Conference, eds. R. A. Laing, \& K. M. Blundell, ASP Conf. Ser., 250, 184

Krichbaum, T. P., Graham, D. A., Bremer, M., et al. 2006, J. Phys. Conf. Ser., 54,328

Kriss, G. A., Davidsen, A. F., Zheng, W., \& Lee, G. 1999, ApJ, 527, 683

Lott, B., Escande, L., Larsson, S., \& Ballet, J. 2012, A\&A, 544, A6

Marscher, A. P. 2013 [arXiv: 1304.2064]
Mattox, J. R., Bertsch, D. L., Chiang, J., et al. 1996, ApJ, 461, 396 Nalewajko, K. 2013, MNRAS, 430, 1324

Nolan, P. L., Abdo, A. A., Ackermann, M., et al. 2012, ApJS, 199, 31

Paltani, S., \& Türler, M. 2003, ApJ, 583, 659

Paltani, S., \& Türler, M. 2005, A\&A, 435, 811

Peterson, B. M., Ferrarese, L., Gilbert, K. M., et al. 2004, ApJ, 613, 682

Pian, E., Urry, C. M., Maraschi, L., et al. 1999, ApJ, 521, 112

Planck Collaboration 2013, A\&A, submitted [arXiv: 1303.5076]

Poutanen, J., \& Stern, B. 2010, ApJ, 717, L118

Rani, B., Gupta, A. C., Strigachev, A., et al. 2010, MNRAS, 404, 1992

Rani, B., Gupta, A. C., Joshi, U. C., Ganesh, S., \& Wiita, P. J. 2011, MNRAS, 413, 2157

Rani, B., Krichbaum, T. P., Fuhrmann, L., et al. 2013a, A\&A, 552, A11

Rani, B., Krichbaum, T. P., Lott, B., Fuhrmann, L., \& Zensus, J. A. 2013b, Adv. Space Res., 51, 2358

Saito, S., Stawarz, Ł., Tanaka, Y. T., et al. 2013, ApJ, 766, L11

Savolainen, T., Wiik, K., Valtaoja, E., \& Tornikoski, M. 2006, A\&A, 446, 71

Savolainen, T., Wiik, K., Valtaoja, E., \& Tornikoski, M. 2008, in Extragalactic Jets: Theory and Observation from Radio to Gamma Ray, eds. T. A. Rector, \& D. S. De Young, ASP Conf. Ser., 386, 451

Savolainen, T., Homan, D. C., Hovatta, T., et al. 2010, A\&A, 512, A24

Schmidt, M. 1963, Nature, 197, 1040

Sikora, M., Błażejowski, M., Begelman, M. C., \& Moderski, R. 2001, ApJ, 554, 1

Sokolov, A., \& Marscher, A. P. 2004, in X-ray Timing 2003: Rossi and Beyond, eds. P. Kaaret, F. K. Lamb, \& J. H. Swank, AIP Conf. Ser., 714, 198

Soldi, S., Türler, M., Paltani, S., et al. 2008, A\&A, 486, 411

Spergel, D. N., Verde, L., Peiris, H. V., et al. 2003, ApJS, 148, 175

Strauss, M. A., Huchra, J. P., Davis, M., et al. 1992, ApJS, 83, 29

Svensson, R. 1987, MNRAS, 227, 403

Swanenburg, B. N., Hermsen, W., Bennett, K., et al. 1978, Nature, 275, 298

Tanaka, Y. T., Stawarz, Ł., Thompson, D. J., et al. 2011, ApJ, 733, 19

Tavecchio, F., \& Ghisellini, G. 2012, MNRAS, submitted [arXiv: 1209.2291]

Tavecchio, F., Ghisellini, G., Bonnoli, G., \& Ghirlanda, G. 2010, MNRAS, 405, L94

Ulrich, M.-H. 1981, Space Sci. Rev., 28, 89

Valtaoja, E., Lähteenmäki, A., Teräsranta, H., \& Lainela, M. 1999, ApJS, 120, 95 